\title{
Ação de herbicidas pré-emergentes no estabelecimento inicial de plântulas de espécies nativas do Cerrado
}

\author{
Action of pre-emergence herbicides in the initial establishment of \\ seedlings of savana's native species
}

Sidnei Roberto de Marchi ${ }^{1}$, Ricardo Fagundes Marques²*, Prissila Pereira dos Santos Araújo², Anney Silva Marques ${ }^{1}$, Rodrigo Marques de Souza ${ }^{1}$

Resumo - A dificuldade de controle das plantas daninhas tem sido considerada como um dos principais entraves ao sucesso dos projetos de recuperação da vegetação em áreas degradadas. O presente trabalho teve o objetivo de avaliar o efeito de diferentes herbicidas pré-emergentes sobre a emergência, sobrevivência e desenvolvimento inicial de plantas das espécies nativas amendoim bravo (Platypodium elegans), mutamba (Guazuma ulmifolia), carobinha (Jacaranda micrantha) e angico cuiabano (Anadenanthera colubrina). $\mathrm{O}$ experimento foi desenvolvido em casa de vegetação no delineamento experimental inteiramente casualizado, com cinco repetições. Os tratamentos foram constituídos pela aplicação em pré-emergência dos seguintes herbicidas e doses: isoxaflutole $\left(75,150\right.$ e $300 \mathrm{~g}$ i.a. ha $\left.{ }^{-1}\right)$, pendimenthalin $\left(500,1000\right.$ e 2000 g i.a. ha $\left.{ }^{-1}\right)$, trifluralin $(600,1200$ e 2400 g i.a. ha-1) e uma testemunha padrão sem a aplicação de herbicidas. O efeito dos herbicidas foi avaliado quanto ao número de plântulas emergidas, número de folhas por plântula, altura de plântulas, diâmetro de plântulas na altura do coleto e matéria seca de raiz e folhas aos 35 dias após a germinação. Com os resultados obtidos foi possível afirmar que todos os herbicidas e doses utilizadas não prejudicaram a emergência e o desenvolvimento inicial das espécies $P$. elegans e $J$. micrantha. As doses de 150 e $300 \mathrm{~g}^{\text {i.a. }}$ ha $^{-1}$ do herbicida isoxaflutole foram não seletivas para a espécie arbórea $G$. ulmifolia. Apenas a dose de $600 \mathrm{~g}$ i.a. ha-1 do herbicida trifluralin apresentou seletividade para a espécie $A$. colubrina.

Palavras-chave: graminicidas, pré-emergência, repovoamento florestal, seletividade

Abstract - The weed control difficulties has been considered one of the most obstacles to success of forest recovery projects at degraded areas. This experiment aimed to evaluate the different pre-emergent herbicides effect on seed emergence, seedling survival and initial development of Brazilian native tree species Platypodium elegans, Guazuma ulmifolia, Anadenanthera colubrine and Jacaranda micrantha. The experiment was conducted under greenhouse conditions in a completely randomized design with five replications. The treatments were constituted by the pre-emergence application of the following herbicides and doses: isoxaflutole $\left(75,150\right.$ and $300 \mathrm{~g}$ a.i. ha $\left.{ }^{-1}\right)$, pendimenthalin $\left(500,1000\right.$ and $2000 \mathrm{~g}^{2}$.i. ha $\left.\mathrm{h}^{-1}\right)$, trifluralin $\left(600,1200\right.$ and $2400 \mathrm{~g}$ a.i. ha $\left.{ }^{-1}\right)$ and untreated herbicide as a check control. The effect of the herbicides was evaluated for the number of seedlings emerged, number of leaves per seedlings, height of seedlings, diameter of stem and dry matter of root and leaves at 35 days after germination. With the results obtained it was possible to affirm that all herbicides and their respective rates were safe to P. elegans and G. ulmifolia trees. Isoxaflutol at 150 and $300 \mathrm{~g}$ a.i. ha ${ }^{-1}$ was not selective to G. ulmifolia specie. Only trifluralin at 600 and $1200 \mathrm{~g}$ a.i. ha ${ }^{-1}$ sowed selectivity to $A$. colubrina specie.

Keywords: grass herbicide, pre-emergence, forest restore, selectivity

Recebido: Julho 16, 2018. Aceito: Janeiro 24, 2019.

${ }^{1}$ Universidade Federal de Mato Grosso - UFMT, Campus do Araguaia, Barra do Garcas, MT, Brasil. E-mail: sidneimarchi.ufmt@gmail.com; anney_markes@hotmail.com; rodrigomarques_agro@hotmail.com

${ }^{2}$ Programa de Pós Graduação em Agronomia, Universidade Federal de Goiás, Regional Jataí, Campus Jatobá, Rodovia BR 364, KM 195, CEP 75801-615, Jataí, GO, Brasil. E-mail: rfmarques94@gmail.com, prissila_araujo@hotmail.com 


\section{Introdução}

A ocupação dos ecossistemas utilizando a supressão vegetal para o desenvolvimento do agronegócio é motivo de grande preocupação ao que se refere à ocupação e desmatamento do Cerrado brasileiro, que vem ocorrendo em diferentes épocas e velocidades (Silva et al., 2015). Levantamentos geográficos apontam que aproximadamente 80 milhões de hectares do Cerrado já passaram por essa transformação (Sano et al., 2010) e, por conta disso, aumentou-se a pressão da sociedade quanto à adoção de políticas preservacionistas para a recomposição da vegetação florestal (Borges et al., 2011).

Atualmente, o ajuste ambiental das propriedades rurais tem sido considerado uma das principais demandas do setor agropecuário e dos órgãos ambientais. Isso se deve ao termo da nova Lei Florestal Brasileira e à implementação do cadastro ambiental rural, no qual a restauração de áreas de preservação permanente (APPs) com uso conflitante da terra e implementação ou adequação da reserva legal será obrigatória (Aguiar et al., 2016).

Porém, a implantação de projetos de recuperação de áreas degradadas utilizando-se de espécies nativas é onerosa devido aos gastos com produção, plantio e manejo de mudas, o que desestimula a recuperação de ambientes naturais em larga escala (Farias et al., 2012). Segundo Rondon Neto et al. (2011) ainda são poucos os conhecimentos científicos referentes à seleção de espécies florestais e as técnicas adequadas para a recuperação das áreas degradadas.

Dentre os problemas encontrados para a recuperação dessas áreas, encontra-se o controle de plantas daninhas no início do processo de regeneração natural ou artificial (Gusmão et al., 2011). O controle químico tem sido o método mais eficiente e rápido para o controle de plantas daninhas, quando comparados às capinas manual e mecânica. A utilização de herbicidas possibilita o manejo dessas espécies invasoras em grades áreas, além da baixa utilização de mão de obra (Moraes Neto et al., 2010).

Com o objetivo de melhorar os métodos de controle de plantas daninhas no reflorestamento, herbicidas com ação seletiva para algumas culturas agrícolas têm sido utilizados de forma empírica (Araldi et al., 2015). Monquero et al. (2011) afirmam que, a fim de utilizar o método químico de controle dessas plantas indesejáveis, são necessários estudos para selecionar herbicidas seletivos às espécies nativas utilizadas nos reflorestamentos. A seletividade dos herbicidas é a base para o sucesso do controle químico das plantas daninhas, sendo considerada uma resposta diferencial de diversas espécies de plantas a determinado ingrediente ativo do herbicida (Rocha et al., 2010).

Herbicidas com conhecida ação seletiva para algumas culturas agrícolas têm sido utilizados em processos de recuperação, embora ainda sejam escassos os estudos dos prejuízos potenciais que a utilização desses produtos possa trazer ao desenvolvimento das espécies nativas (Brancalion et al., 2009). Salienta-se que os poucos estudos encontrados abordam sobre a seletividade de herbicidas em espécies nativas florestais aplicados em pós-emergência.

$\mathrm{O}$ amendoim bravo (Platypodium elegans), a mutamba (Guazuma ulmifolia), a carobinha (Jacaranda micrantha) e o angico cuiabano (Anadenanthera colubrina) estão entre as principais espécies nativas recomendadas na recomposição da vegetação em áreas do Cerrado (Campos Filho, 2009). Porém, são escassas as informações sobre seletivos para estas espécies. Diante disso, o presente trabalho teve o objetivo de verificar a seletividade de diferentes doses dos herbicidas isoxaflutole, pendimethalin e trifluralin aplicados na pré-emergência das espécies arbóreas amendoim bravo, mutamba, angico cuiabano e carobinha.

\section{Material e métodos}

O experimento foi conduzido em condições de casa-de-vegetação localizada na Universidade Federal de Mato Grosso - Campus do Araguaia, 
cujas coordenadas geográficas são $15^{\circ} 52$ '29' S e $52^{\circ} 18 ' 35$ " O. O substrato utilizado no experimento foi coletado na camada arável de um Latossolo Vermelho Amarelo distrófico (Santos et al., 2013), onde amostras compostas foram coletadas e enviadas para análise. Suas características químicas e físicas estão apresentadas a seguir: $\mathrm{pH}$ em $\mathrm{CaCl}_{2}$ de 4,8; 22,0 $\mathrm{g} \mathrm{dm}^{-3}$ de matéria orgânica; 4,0 $\mathrm{mg} \mathrm{dm}^{-3}$ de $\mathrm{P}$ resina; $\mathrm{V}$ de 44,6\%; e teores de $\mathrm{K}, \mathrm{Ca}, \mathrm{Mg}$ e $\mathrm{H}+\mathrm{AL}$ de 3,1; 18,0; 6,0 e 34,0 mmolc $\mathrm{dm}^{-3}$, respectivamente; $706 \mathrm{~g} \mathrm{~kg}^{-1}$ de areia, $85 \mathrm{~g} \mathrm{~kg}^{-1}$ de silte e $209 \mathrm{~g} \mathrm{~kg}^{-1}$ de argila, caracterizando-o como sendo de textura franco arenosa. Não foi realizada nenhuma correção quanto à fertilidade e acidez do solo para simular as condições ambientais naturais das áreas de recuperação florestal. O substrato foi acondicionado em vasos de plástico com capacidade para $1,0 \mathrm{~kg}$.

As sementes das espécies arbóreas utilizadas (Tabela 1) foram fornecidas pela organização não governamental Instituto Socioambiental (ISA) e passaram por tratamentos pré-germinativos para evitar a desuniformidade na emergência das plântulas (Mori et al., 2012). A semeadura foi realizada diretamente no vaso em quantidade suficiente para garantir a germinação de aproximadamente três plantas por vaso, sendo que cada espécie foi estudada individualmente e os herbicidas aplicados logo após a semeadura. A irrigação foi realizada diariamente em quantidade suficiente para manter a umidade do solo sem que houvesse saturação da capacidade de campo e consequente perda de água por escorrimento no vaso.

Adotou-se o delineamento experimental inteiramente casualizado, com cinco repetições, onde cada vaso foi considerado uma unidade experimental. Os tratamentos foram constituídos pelo herbicida isoxaflutole (Fordor $750 \mathrm{WG}$,
$750 \mathrm{~g} \mathrm{~kg}^{-1}$ de i.a., WG, Bayer ${ }^{\circledR}$ ) aplicado nas doses de 75, 150 e 300 g i.a. ha ${ }^{-1}$; pendimethalin (Herbadox 400 EC, $400 \mathrm{~g} \mathrm{~L}^{-1}$ de i.a., EC, $\mathrm{BASF}^{\circledR}$ ) aplicado nas doses de 500, 1000 e 2000 g i.a. ha ${ }^{-1}$ e pelo herbicida trifluralin (Premerlin 600 EC, $600 \mathrm{~g} \mathrm{~L}^{-1}$ de i.a., EC, Adama ${ }^{\circledR}$ ) aplicado nas doses de 600, 1200 e 2400 g i.a. ha ${ }^{-1}$, além de uma testemunha padrão sem a aplicação de herbicidas. As doses dos herbicidas corresponderam à uma, duas e quatro vezes a recomendação mínima de rótulo de cada produto, conforme proposto por Brancalion et al. (2009).

Os tratamentos herbicidas foram aplicados em pré-emergência após a semeadura, com o auxílio de pulverizador costal à pressão constante por $\mathrm{CO}_{2}$, contendo barra de pulverização munida de quatro pontas do tipo leque $110015 \mathrm{VS}$, espaçadas $50 \mathrm{~cm}$ entre si, distantes $50 \mathrm{~cm}$ do alvo e calibrada a $35 \mathrm{lbpol}^{2}$ de modo a se obter consumo de calda equivalente a $200 \mathrm{~L} \mathrm{ha}^{-1}$.

A emergência foi avaliada diariamente até o momento em que não houve mais o surgimento de novas plântulas, o que correspondeu a um período de 35 dias após a semeadura. As plântulas foram avaliadas ao final do período experimental quanto ao número por vaso, número de folhas, altura do caule principal e diâmetro de coleto ao nível do solo.

A parte aérea das plântulas foi cortada e todo o material obtido foi acondicionado em sacos de papel. A seguir, as raízes foram cuidadosamente separadas do solo com o auxílio de jatos de água e todo o sistema radicular também acondicionado em sacos de papel. As amostras da parte aérea e do sistema radicular foram mantidas em estufa com circulação forçada de ar e temperatura constante a $60{ }^{\circ} \mathrm{C}$ por 72 horas, sendo depois obtida a matéria seca das frações com o auxílio de balança com

Tabela 1. Relação das espécies nativas utilizadas no experimento e peso de 1000 sementes.

\begin{tabular}{lccr}
\hline \multicolumn{1}{c}{ Espécie } & Família & Nome vulgar & 1000 sementes (g) \\
\hline Platypodium elegans & Fabaceae & Amendoim bravo & 166,70 \\
Guazuma ulmifolia & Malvaceae & Mutamba & 7,40 \\
Jacaranda micranta & Bignoniaceae & Carobinha & 30,30 \\
Anadenanthera colubrina & Fabaceae & Angico cuiabano & 133,30 \\
\hline
\end{tabular}


precisão de 0,001 gramas e calculada a média de matéria seca de parte aérea e do sistema radicular em função do número de plântulas emergidas.

Os valores obtidos para todas as variáveis foram transformados em raiz quadrada de $\mathrm{X}+1$ e os resultados submetidos a análise de variância pelo teste $\mathrm{F}$ com o auxílio do programa estatístico AgroEstat (Barbosa e Maldonado Junior, 2015), sendo que as médias dos tratamentos foram comparadas pelo teste de Scott-Knott $(\mathrm{P}>0,05)$.

\section{Resultados e discussão}

Não foram observados efeitos deletérios dos herbicidas isoxaflutole, pendimenthalin e trifluralin para as variáveis: número de plântulas, número de folhas, altura, diâmetro de coleto, matéria seca de raiz e de parte aérea acumulada pela espécie P. elegans, independentemente da dose utilizada (Tabela 2).

O fruto de $P$. elegans é do tipo sâmara e sua principal característica é apresentar um núcleo seminífero envolto por pericarpo rígido e contornado por alas coriáceas vascularizadas, indicando que as sementes dessa espécie apresentam alta resistência as condições desfavoráveis para germinação (Pacheco et al., 2007). Assim, é possível inferir que os herbicidas aplicados em pré-emergência não afetaram a viabilidade das sementes de $P$. elegans devido à rigidez do pericarpo conferindo germinação e emergência normal para a espécie.

O herbicida trifluralin aplicado nas doses 600,1200 e 2400 g i.a. ha ${ }^{-1}$ foi seletivo para espécie G. ulmifolia, pois não afetou nenhuma das variáveis avaliadas. Todavia, os demais herbicidas e suas respectivas doses proporcionaram redução no número e altura de plântulasTabela 3).

Destaca-se que o maior número de folhas (3,37 folhas) em plântulas de G. ulmifolia foi proporcionado quando utilizado o herbicida isoxaflutole na dose de $75 \mathrm{~g}$ i.a. ha-1 ${ }^{-1}$ O pendimethalin quando aplicado na dose de 500 e $1000 \mathrm{~g}$ i.a. $\mathrm{ha}^{-1} \mathrm{e}$ a trifluralin nas doses de 600, 1200 e $2400 \mathrm{~g}$ i.a. ha ${ }^{-1}$ não diferiram da testemunha quanto ao número de folhas por plântulas de G. ulmifolia (Tabela 3).

Verificou-se também que, independentemente da dose utilizada do herbicida trifluralin e na dose de 500 g i.a. ha-1 de pendimethalin, os valores de diâmetro de coleto foram semelhantes à testemunha. Diferentemente, o isoxaflutole a 75,150 e $300 \mathrm{~g}$ i.a. ha ${ }^{-1}$ e o pendimethalin a 1000 e 2000 g i.a. ha ${ }^{-1}$ proporcionaram reduções significativas de $13,3 \%, 33,3 \%, 33,3 \%, 8,0 \%$ e

Tabela 2. Efeito de herbicidas e doses sobre o número de plântulas, número de folhas, altura de plântulas $(\mathrm{cm})$, diâmetro do coleto $(\mathrm{mm})$ e matéria seca $(\mathrm{g})$ de raiz e parte aérea de $P$. elegans.

\begin{tabular}{|c|c|c|c|c|c|c|}
\hline \multirow{2}{*}{$\begin{array}{c}\text { Tratamento } \\
\left(\mathrm{g} \text { i.a. } \text { ha }^{-1}\right) \\
\end{array}$} & \multicolumn{2}{|c|}{ Número } & \multirow{2}{*}{$\begin{array}{l}\text { Altura } \\
(\mathrm{cm})\end{array}$} & \multirow{2}{*}{$\begin{array}{c}\text { Diâmetro } \\
\text { (mm) }\end{array}$} & \multicolumn{2}{|c|}{ Matéria seca (g) } \\
\hline & Plântulas & Folhas & & & Raiz & Parte aérea \\
\hline Isoxaflutole (75) & 1,94 & 2,42 & 3,94 & 1,09 & 1,07 & 1,17 \\
\hline Isoxaflutole (150) & 2,13 & 2,38 & 3,32 & 1,07 & 1,07 & 1,19 \\
\hline Isoxaflutole (300) & 1,82 & 2,26 & 3,40 & 1,08 & 1,03 & 1,15 \\
\hline Pendimenthalin (500) & 1,86 & 2,80 & 3,74 & 1,10 & 1,12 & 1,20 \\
\hline Pendimenthalin (1000) & 2,13 & 2,59 & 3,95 & 1,09 & 1,08 & 1,18 \\
\hline Pendimenthalin (2000) & 2,09 & 2,62 & 4,01 & 1,11 & 1,11 & 1,19 \\
\hline Trifluralin $(600)$ & 1,83 & 2,63 & 3,58 & 1,09 & 1,08 & 1,50 \\
\hline Trifluralin (1200) & 1,72 & 2,32 & 3,28 & 1,09 & 1,09 & 1,14 \\
\hline Trifluralin (2400) & 1,84 & 2,43 & 3,86 & 1,10 & 1,11 & 1,21 \\
\hline Testemunha & 2,28 & 2,61 & 3,71 & 1,07 & 1,09 & 1,15 \\
\hline F Tratamentos & $1,86^{\mathrm{NS}}$ & $0,99^{\mathrm{NS}}$ & $1,06^{\mathrm{NS}}$ & $1,19^{\mathrm{NS}}$ & $0,99^{\mathrm{NS}}$ & $2,45^{\mathrm{NS}}$ \\
\hline C.V. $(\%)$ & 15,0 & 15,70 & 16,0 & 2,30 & 5,50 & 5,20 \\
\hline
\end{tabular}

Dados transformados em raiz quadrada de $\mathrm{x}+1{ }^{\mathrm{NS}}=$ Não significativo; g i.a. ha $^{-1}=$ gramas de ingrediente ativo por hectare; C.V. $(\%)=$ Coeficiente de variação. 
Tabela 3. Efeito de herbicidas e doses sobre o número de plântulas, número de folhas, altura de plântulas $(\mathrm{cm})$, diâmetro do coleto $(\mathrm{mm})$ e matéria seca $(\mathrm{g})$ de raiz e parte aérea de G. ulmifolia.

\begin{tabular}{|c|c|c|c|c|c|c|}
\hline \multirow{2}{*}{$\begin{array}{l}\text { Tratamento } \\
\left.\text { (g i.a. ha }^{-1}\right)\end{array}$} & \multicolumn{2}{|c|}{ Número } & \multirow{2}{*}{$\begin{array}{l}\text { Altura } \\
(\mathrm{cm})\end{array}$} & \multirow{2}{*}{$\begin{array}{l}\text { Diâmetro } \\
\text { (mm) }\end{array}$} & \multicolumn{2}{|c|}{ Matéria seca (g) } \\
\hline & Plântulas & Folhas & & & Raiz & Parte aérea \\
\hline Isoxaflutole (75) & $1,57 \mathrm{c}$ & $3,37 \mathrm{a}$ & $1,30 \mathrm{~b}$ & $1,30 \mathrm{~b}$ & $1,61 \mathrm{a}$ & $1,56 \mathrm{a}$ \\
\hline Isoxaflutole (150) & $1,00 \mathrm{~d}$ & $1,00 \mathrm{~d}$ & $1,00 \mathrm{c}$ & $1,00 \mathrm{~d}$ & $1,00 \mathrm{c}$ & $1,00 \mathrm{c}$ \\
\hline Isoxaflutole (300) & $1,00 \mathrm{~d}$ & $1,00 \mathrm{~d}$ & $1,00 \mathrm{c}$ & $1,00 \mathrm{~d}$ & $1,00 \mathrm{c}$ & $1,00 \mathrm{c}$ \\
\hline Pendimenthalin (500) & $2,32 \mathrm{~b}$ & $2,14 \mathrm{~b}$ & $1,47 \mathrm{~b}$ & $1,44 \mathrm{a}$ & $1,54 \mathrm{a}$ & $1,62 \mathrm{a}$ \\
\hline Pendimenthalin (1000) & $2,13 \mathrm{~b}$ & $2,16 \mathrm{~b}$ & $1,45 \mathrm{~b}$ & $1,38 \mathrm{~b}$ & $1,54 \mathrm{a}$ & $1,48 \mathrm{a}$ \\
\hline Pendimenthalin (2000) & $1,17 \mathrm{~d}$ & $1,44 \mathrm{c}$ & $1,16 \mathrm{c}$ & $1,16 \mathrm{c}$ & $1,21 \mathrm{~b}$ & $1,27 \mathrm{~b}$ \\
\hline Trifluralin (600) & $2,65 \mathrm{a}$ & $2,49 \mathrm{~b}$ & $1,78 \mathrm{a}$ & $1,50 \mathrm{a}$ & $1,52 \mathrm{a}$ & $1,53 \mathrm{a}$ \\
\hline Trifluralin (1200) & $3,02 \mathrm{a}$ & $2,48 \mathrm{~b}$ & $1,98 \mathrm{a}$ & $1,49 \mathrm{a}$ & $1,56 \mathrm{a}$ & $1,50 \mathrm{a}$ \\
\hline Trifluralin (2400) & $3,09 \mathrm{a}$ & $2,44 \mathrm{~b}$ & $1,91 \mathrm{a}$ & $1,60 \mathrm{a}$ & $1,53 \mathrm{a}$ & $1,52 \mathrm{a}$ \\
\hline Testemunha & $3,01 \mathrm{a}$ & $2,40 \mathrm{~b}$ & $1,83 \mathrm{a}$ & $1,50 \mathrm{a}$ & $1,58 \mathrm{a}$ & $1,52 \mathrm{a}$ \\
\hline F Tratamentos & $37,13^{* *}$ & $26,73^{* *}$ & $17,26^{* *}$ & $18,14^{* *}$ & $21,29 * *$ & $13,93 * *$ \\
\hline C.V. $(\%)$ & 15,0 & 15,40 & 13,40 & 8,50 & 8,30 & 9,80 \\
\hline
\end{tabular}

Dados transformados em raiz quadrada de $\mathrm{x}+1{ }^{* *}$ Significativo ao nível de $1 \%$ de probabilidade; $\mathrm{g}$ i.a. ha $^{-1}=$ gramas de ingrediente ativo por hectare; C.V. $(\%)=$ Coeficiente de variação; Médias seguidas de mesma letra na coluna não diferem estatisticamente entre si pelo teste de Scott-Knott a 5\% de probabilidade.

$22,7 \%$, respectivamente, no diâmetro de coleto da espécie G. ulmifolia (Tabela 3).

Embora tenham sido observados efeitos sobre algumas variáveis avaliadas em plântulas de G. ulmifolia, estes pouco influenciaram o acúmulo de matéria seca de raiz e parte aérea. Apenas o herbicida isoxaflutole, nas doses de 150 e 300 g i.a. ha-1, e o pendimethalin, na maior dose de $2000 \mathrm{~g}$ i.a. ha ${ }^{-1}$, reduziram a quantidade de matéria seca de raiz e parte aérea produzida pela G. ulmifolia (Tabela 3), o que evidencia o potencial risco de uso do isoxaflutole e pendimethalin e respectivas doses nas ações de recuperação de áreas degradadas e em plantios comerciais com essa arbórea nativa.

Assim, pode-se afirmar que a espécie G. ulmifolia é tolerante ao herbicida isoxaflutole aplicado na dose de $75 \mathrm{~g}$ i.a ha ${ }^{-1} \mathrm{e}$ ao pendimethalin aplicado nas doses de 500 e $1000 \mathrm{~g}$ i.a. ha-1 ${ }^{-1}$. Nesse caso, a tolerância pode estar relacionada à metabolização rápida das moléculas até formas não tóxicas, o que evita a morte das plantas (Brancalion et al., 2009). Especificamente para isoxaflutole, já foi observado em cana-de-açúcar e milho que a diquetonitrila, molécula que possui ação herbicida, é convertida em metabólito ácido benzoico, sem ação herbicida e que se converte posteriormente em gás carbônico (Oliveira Júnior et al., 2006).

Dessa forma, a ausência de redução da matéria seca de raízes e parte aérea das plântulas é indício de que a seletividade ou tolerância aos herbicidas testados em G. ulmifolia, possivelmente, se devem à metabolização dos princípios ativos, o que reduz progressivamente a ação fitotóxica dos mesmos e permite a continuidade do desenvolvimento normal das plantas.

Os herbicidas isoxaflutole e pendimenthalin apresentaram efeito negativo sobre o número de plântulas emergidas de J. micranta. O mesmo efeito foi observado quando a trifluralin foi utilizada nas doses de 1200 e 2400 g i.a. ha ${ }^{-1}$, uma vez que o número de plântulas emergidas variou entre 1,17 e 1,56 plantas por vaso em comparação ao valor de 1,99 plântulas na testemunha (Tabela 4). Salienta-se que não foram observados efeitos negativos causados pelos herbicidas sobre o número de folhas, a altura de plântulas, o diâmetro de coleto, a matéria seca de raiz e parte aérea para a espécie J. micrantha (Tabela 4).

Existem poucos estudos do comportamento inicial em termos de germinação e desenvolvimento de plântulas de J. micranta em qualquer condição 
Tabela 4. Efeito de herbicidas e doses sobre o número de plântulas, número de folhas, altura de plântulas $(\mathrm{cm})$, diâmetro do coleto $(\mathrm{mm})$ e matéria seca $(\mathrm{g})$ de raiz e parte aérea de J. micrantha.

\begin{tabular}{|c|c|c|c|c|c|c|}
\hline \multirow{2}{*}{$\begin{array}{l}\text { Tratamento } \\
\left(\text { g i.a. }^{-1} \mathbf{a}^{-1}\right.\end{array}$} & \multicolumn{2}{|c|}{ Número } & \multirow{2}{*}{$\begin{array}{l}\text { Altura } \\
(\mathrm{cm})\end{array}$} & \multirow{2}{*}{$\begin{array}{c}\text { Diâmetro } \\
(\mathbf{m m})\end{array}$} & \multicolumn{2}{|c|}{ Matéria seca (g) } \\
\hline & Plântulas & Folhas & & & Raiz & Parte aérea \\
\hline Isoxaflutole (75) & $1,38 \mathrm{~b}$ & 2,58 & 1,84 & 1,04 & 1,06 & 1,04 \\
\hline Isoxaflutole (150) & $1,17 \mathrm{~b}$ & 2,15 & 1,61 & 1,02 & 1,04 & 1,04 \\
\hline Isoxaflutole (300) & $1,28 \mathrm{~b}$ & 2,00 & 1,47 & 1,01 & 1,01 & 1,01 \\
\hline Pendimenthalin (500) & $1,39 \mathrm{~b}$ & 3,14 & 2,30 & 1,06 & 1,28 & 1,11 \\
\hline Pendimenthalin (1000) & $1,23 \mathrm{~b}$ & 2,15 & 1,64 & 1,02 & 1,05 & 1,05 \\
\hline Pendimenthalin (2000) & $1,48 \mathrm{~b}$ & 3,77 & 2,47 & 1,06 & 1,13 & 1,10 \\
\hline Trifluralin (600) & $1,72 \mathrm{a}$ & 2,08 & 1,59 & 1,03 & 1,07 & 1,02 \\
\hline Trifluralin (1200) & $1,56 \mathrm{~b}$ & 3,04 & 2,15 & 1,05 & 1,14 & 1,05 \\
\hline Triffuralin (2400) & $1,23 \mathrm{~b}$ & 2,72 & 1,91 & 1,03 & 1,10 & 1,05 \\
\hline Testemunha & $1,99 \mathrm{a}$ & 3,42 & 2,52 & 1,06 & 1,13 & 1,11 \\
\hline F Tratamentos & $2,26^{*}$ & $1,14^{\mathrm{NS}}$ & $1,35^{\mathrm{NS}}$ & $1,88^{\mathrm{NS}}$ & $2,03^{\mathrm{NS}}$ & $1,95^{\mathrm{NS}}$ \\
\hline C.V.(\%) & 26,60 & 48,11 & 38,0 & 3,00 & 10,70 & 5,30 \\
\hline
\end{tabular}

Dados transformados em raiz quadrada de $\mathrm{x}+1 ; *$ Significativo ao nível de $5 \%$ de probabilidade; ${ }^{\text {NS }}=$ Não significativo; g i.a. ha $^{-1}=$ gramas de ingrediente ativo por hectare; C.V. $(\%)=$ Coeficiente de variação; Médias seguidas de mesma letra na coluna não diferem estatisticamente entre si pelo teste de Scott-Knott a 5\% de probabilidade.

de ambiente e substrato (Bovolini et al., 2015). Porém, ao avaliarem o uso de espécies florestais para diminuição do resíduo de clomazone, no ambiente, Cabral et al. (2017) observaram que a altura das plantas de Jacaranda puberula mostraram aumento de $12 \%$ em média pela aplicação do herbicida. Fiore et al. (2016) observaram que mudas dessa espécie apresentaram aumento significativo de $9 \%$ quando aplicado o herbicida atrazine e $14,75 \%$ quando aplicado 2,4-D no parâmetro altura de plantas. Constata-se assim que além da variação de respostas entre diferentes famílias e espécies vegetais, a fitotoxicidade de diferentes herbicidas a espécies arbóreas nativas também pode ser observada dentro de uma mesma espécie, como consequência da variabilidade genética (Ferreira et al., 2005).

A. colubrina foi a espécie de maior sensibilidade aos tratamentos herbicidas utilizados. Apenas o herbicida trifluralin utilizado na menor dose de 600 g i.a. ha ${ }^{-1}$ apresentou seletividade a esta espécie, uma vez que as variáveis número de plântulas por vaso, número de folhas por plântula, altura e diâmetro de coleto das plântulas e matéria seca de parte aérea não diferiram da testemunha (Tabela 5).
A espécie $A$. colubrina pode ser recomendada para o plantio em ambientes degradados em virtude da alta sobrevivência e crescimento inicial das plântulas. Porém, condições adversas podem comprometer a viabilidade dessas mudas por serem extremamente sensíveis a mudanças (Nunes et al., 2015). Essa sensibilidade das mudas e o fato dessa espécie apresentar sementes sem tegumento que possa garantir dormência e resistência a situações estressantes podem explicar o fato de apenas a dose de 600 g i.a. ha-1 do herbicida trifluralin apresentar seletividade para a espécie $A$. colubrina.

Destaca-se que a ação dos herbicidas foi diferenciada de espécie para espécie, uma vez que $P$. elegans e $J$. micranta se mostram mais tolerantes a todas as doses dos herbicidas utilizados quando comparados às outras duas espécies estudas. Segundo Duarte et al. (2006) a menor sensibilidade aos herbicidas de espécies nativas pode estar também relacionada à relação absorção/metabolização do ingrediente ativo do herbicida, onde quando maior a relação entre eles mais prejudicial é sua ação na planta.

Quando avaliada a ação dos herbicidas em diferentes espécies do mesmo gênero, foram 
Tabela 5. Efeito de herbicidas e doses sobre o número de plântulas, número de folhas, altura de plântulas $(\mathrm{cm})$, diâmetro do coleto $(\mathrm{mm})$ e matéria seca $(\mathrm{g})$ de raiz e parte aérea de $A$. colubrina.

\begin{tabular}{|c|c|c|c|c|c|c|}
\hline \multirow{2}{*}{$\begin{array}{l}\text { Tratamento } \\
\left.\text { (g i.a. ha } \mathbf{h}^{-1}\right)\end{array}$} & \multicolumn{2}{|c|}{ Número } & \multirow{2}{*}{$\begin{array}{c}\text { Altura } \\
\text { (cm) }\end{array}$} & \multirow{2}{*}{$\begin{array}{c}\text { Diâmetro } \\
\text { (mm) }\end{array}$} & \multicolumn{2}{|c|}{ Matéria seca (g) } \\
\hline & Plântulas & Folhas & & & Raiz & Parte aérea \\
\hline Isoxaflutole (75) & $1,00 \mathrm{~b}$ & $1,00 \mathrm{~b}$ & $1,00 \mathrm{~b}$ & $1,00 \mathrm{~b}$ & $1,00 \mathrm{~b}$ & $1,00 \mathrm{c}$ \\
\hline Isoxaflutole (150) & $1,00 \mathrm{~b}$ & $1,00 \mathrm{~b}$ & $1,00 \mathrm{~b}$ & $1,00 \mathrm{~b}$ & $1,00 \mathrm{~b}$ & $1,00 \mathrm{c}$ \\
\hline Isoxaflutole (300) & $1,00 \mathrm{~b}$ & $1,00 \mathrm{~b}$ & $1,00 \mathrm{~b}$ & $1,00 \mathrm{~b}$ & $1,00 \mathrm{~b}$ & $1,00 \mathrm{c}$ \\
\hline Pendimenthalin (500) & $1,15 \mathrm{~b}$ & $1,22 \mathrm{~b}$ & $1,00 \mathrm{~b}$ & $1,02 \mathrm{~b}$ & $1,02 \mathrm{~b}$ & $1,01 \mathrm{c}$ \\
\hline Pendimenthalin (1000) & $1,00 \mathrm{~b}$ & $1,00 \mathrm{~b}$ & $1,00 \mathrm{~b}$ & $1,00 \mathrm{~b}$ & $1,00 \mathrm{~b}$ & $1,00 \mathrm{c}$ \\
\hline Pendimenthalin (2000) & $1,00 \mathrm{~b}$ & $1,00 \mathrm{~b}$ & $1,38 \mathrm{~b}$ & $1,00 \mathrm{~b}$ & $1,00 \mathrm{~b}$ & $1,00 \mathrm{c}$ \\
\hline Triffuralin $(600)$ & $1,41 \mathrm{a}$ & $2,09 \mathrm{a}$ & $2,93 \mathrm{a}$ & $1,10 \mathrm{a}$ & $1,08 \mathrm{~b}$ & $1,08 \mathrm{a}$ \\
\hline Trifluralin (1200) & $1,17 \mathrm{~b}$ & $1,40 \mathrm{~b}$ & $1,68 \mathrm{~b}$ & $1,03 \mathrm{~b}$ & $1,06 \mathrm{~b}$ & $1,03 \mathrm{~b}$ \\
\hline Triffuralin (2400) & $1,08 \mathrm{~b}$ & $1,15 \mathrm{~b}$ & $1,20 \mathrm{~b}$ & $1,01 \mathrm{~b}$ & $1,01 \mathrm{~b}$ & $1,01 \mathrm{c}$ \\
\hline Testemunha & $1,54 \mathrm{a}$ & $1,84 \mathrm{a}$ & $2,69 \mathrm{a}$ & $1,08 \mathrm{a}$ & $1,18 \mathrm{a}$ & $1,07 \mathrm{a}$ \\
\hline F Tratamentos & $8,41^{* *}$ & $10,98 * *$ & $14,41 * *$ & $17,50^{* *}$ & $4,52 * *$ & $15,35 * *$ \\
\hline C.V. $(\%)$ & 13,20 & 20,90 & 29,10 & 1,90 & 6,10 & 1,80 \\
\hline
\end{tabular}

Dados transformados em raiz quadrada de $\mathrm{x}+1{ }^{* *}$ Significativo ao nível de $1 \%$ de probabilidade; $\mathrm{g}$ i.a. ha $^{-1}=$ gramas de ingrediente ativo por hectare; C.V. $(\%)=$ Coeficiente de variação; Médias seguidas de mesma letra na coluna não diferem estatisticamente entre si pelo teste de Scott-Knott a 5\% de probabilidade.

encontradas diferenças quanto à tolerância. Esse fato ressalta a dificuldade de generalização de métodos de controle de plantas daninhas em reflorestamentos com elevada diversidade de espécies nativas, o que justifica a necessidade de proteção adicional de espécies de algumas famílias e gêneros vegetais, caso se opte pelo uso desses herbicidas (Brancalion et al., 2009).

De acordo com os resultados obtidos, pode-se inferir que os herbicidas estudados, se utilizados com critério, apresentam potencial de uso no repovoamento de APPs, através de modelos que utilizam o consórcio com as espécies nativas $P$. elegans, e $J$. micrantha representando uma alternativa eficiente para o manejo de gramíneas. No entanto, em decorrência da toxicidade dos herbicidas no meio ambiente, sua utilização deve ser feita com técnica e responsabilidade, pois podem prejudicar a fauna e flora (Ferreira et al., 2005).

\section{Conclusões}

Todos os herbicidas e doses utilizadas não prejudicaram a emergência e o desenvolvimento inicial das espécies $P$. elegans e $J$. micrantha.
As doses de 150 e 300 g i.a. ha ${ }^{-1}$ do herbicida isoxaflutole foram não seletivas para a espécie arbórea G. ulmifolia. Apenas a dose de 600 g i.a. ha ${ }^{-1}$ do herbicida trifluralin apresentou seletividade para a espécie $A$. colubrina.

\section{Referências}

Aguiar, L.M.; Santos, J.B.; Costa, V.A.; Brito, L.A.; Ferreira, E.A.; Pereira, I.M.; et al. Herbicide tolerance and water use effciency in forest species used in degraded áreas recovery programs. Bosque (Valdivia), v.37, n.3, p.493-500, 2016. http:// dx.doi.org/10.4067/S0717-92002016000300006.

Araldi, R.; Corniani, N.; Tropaldi, L.; Girotto, M.; Belapart, D.; Simões, P.S.; Velini, E.D. Chlorophyll fluorescence in guanandi tree (Calophyllum brasiliense) after herbicide application. Planta Daninha, v.33, n.1, p.77-82, 2015. http://dx.doi. org/10.1590/S0100-83582015000100009.

Barbosa, J.C.; Maldonado Junior, W. Experimentação agronômica \& AgroEstat: sistemas para análises estatísticas e ensaios agronômicos. Jaboticabal: Gráfica Multipress, 2015. 396p. 
Borges, L.A.C.; Rezende, J.L.P.; Pereira, J.A.A.; Coelho Junior, L.M.; Barros, D.A. Áreas de preservação permanente na legislação ambiental brasileira. Ciência Rural, v.41, n.7, p.12021210, 2011. http://dx.doi.org/10.1590/S010384782011000700016 .

Bovolini, M.P.; Maciel, C.G.; Brum, D.; Muniz, M.F.B. Influência de temperatura e substrato na germinação e no vigor de sementes de Jacaranda micrantha Cham. Revista de Ciências Agroveterinárias, v.14, n.3, p.203-209, 2015. http://dx.doi.org/10.5965/223811711432015203.

Brancalion, P.H.S.; Isernhagen, I.; Machado, R.P.; Christoffoleti, P.J.; Rodrigues, R.R. Seletividade dos herbicidas setoxidim, isoxaflutol e bentazona espécies arbóreas nativas. Pesquisa Agropecuária Brasileira, v.44, n.3, p.251-257, 2009. http:// dx.doi.org/10.1590/S0100-204X2009000300005.

Cabral, C.M.; Santos, J.B.; Ferreira, E.A.; Machado, V.M.; Pereira, I.M.; Silva, D.V.; Souza, M.F. Tolerance to the herbicide clomazone and potential for changes of forest species. Bioscience Journal, v.33, n.4, p.897-904, 2017. http://dx.doi. org/10.14393/BJ-v33n4a2017-36750.

Campos Filho, E.M. Plante as árvores do Xingu e Araguaia: guia de identificação. São Paulo: Instituto Socioambiental, 2009. 297p. v.3.

Duarte, N.F.; Karam, D.; Sá, N.; Cruz, M.B.; Scotti, M.R.M. Seletividade de herbicidas sobre Myracrodruon urundeuva (aroeira). Planta Daninha, v.24, n.2, p.329-337, 2006. http:// dx.doi.org/10.1590/S0100-83582006000200016.

Farias, C.C.M.; Rondon Neto, R.M.; Yamashita, O.M.; Farias, C.B.M. Efeitos de subdoses de glyphosate em plantas jovens de seringueira (Hevea brasiliensis Aubl.). Revista Brasileira de Herbicidas, v.11, n.1, p.119-125, 2012. http:// dx.doi.org/10.7824/rbh.v11i1.116.

Ferreira, R.A.; Davide, A.C.; Alcântara, E.F.; Motta, M.S. Efeito de herbicidas de pré-emergência sobre o desenvolvimento inicial de espécies arbóreas.
Revista Brasileira de Herbicidas, v.4, n.1, p.133, 2005. http://dx.doi.org/10.7824/rbh.v4i1.17.

Fiore, R.A.; Santos, J.B.; Ferreira, E.A.; Cabral, C.M.; Pereira, I.M. Growth and nutritional analysis of tree species in contaminated substrate by leachable herbicides. Revista Árvore, v.40, n.4, p.585-594, 2016. http://dx.doi.org/10.1590/010067622016000400002 .

Gusmão, G.A.; Rondon Neto, R.M.; Yamashita, O.M. Deriva simulada de glyphosate em plantas jovens de jenipapo (Genipa americana L.). Revista Brasileira de Herbicidas, v.10, n.1, p.13-19, 2011. http://dx.doi.org/10.7824/rbh.v10i1.91.

Monquero, P.A.; Penha, A.S.; Orzari, I.; Hirata, A.C.S. Seletividade de herbicidas a mudas das espécies nativas Acacia polyphylla, Enterolobium contortisiliquum (Fabaceae), Ceiba speciosa e Luehea divaricata (Malvaceae). Planta Daninha, v.29, n.1, p.159, 2011. http://dx.doi.org/10.1590/ S0100-83582011000100018.

Moraes Neto, S.P.; Poggiani, F.; Rodrigues, R.R.; Christoffoleti, P.J. Efeito do controle de gramíneas no crescimento de quatro espécies arbóreas no fragmento de floresta secundária. Planaltina: Embrapa Cerrados, 2010.23p. (Boletim de Pesquisa e Desenvolvimento, 289).

Mori, E.S.; Piña-Rodrigues, F.C.M.; Freitas, N.P. Sementes florestais: guia para germinação de 100 espécies nativas. 1. ed. São Paulo: Instituto Refloresta, 2012. 159p.

Nunes, Y.R.F.; Fagundes, N.C.A.; Veloso, M.D.M.; Gonzaga, A.P.D.; Domingues, E.B.S.; Almeida, H.S.; et al. Sobrevivência e crescimento de sete espécies arbóreas nativas em uma área degradada de floresta estacional decidual, norte de Minas Gerais. Revista Árvore, v.39, n.5, p.801-810, 2015. http:// dx.doi.org/10.1590/0100-67622015000500003.

Oliveira Júnior, R.S.; Marchiori Júnior, O.; Constantin, J.; Inoue, M.H. Influência do período de restrição hídrica na atividade residual de isoxaflutole no solo. Planta Daninha, v.24, 
n.4, p.733-740, 2006. http://dx.doi.org/10.1590/ S0100-83582006000400014.

Pacheco, M.V.; Matos, V.P.; Barbosa, M.D.; Ferreira, R.L.C.; Passos, M.A.A. Germinação de sementes de Platypodium elegans Vog. submetidas a diferentes tratamentos prégerminativos e substratos. Revista Brasileira de Engenharia Agrícola e Ambiental, v.11, n.5, p.497-501, 2007. http://dx.doi.org/10.1590/ S1415-43662007000500008.

Rocha, P.R.R.; Silva, A.F.; Faria, A.T.; Galon, L.; Ferreira, E.A.; Felipe, R.S.; et al. Seletividade de herbicidas pré-emergentes ao pinhão-manso (Jatropha curcas). Planta Daninha, v.28, n.4, p.801-806, 2010. http://dx.doi.org/10.1590/ S0100-83582010000400013.

Rondon Neto, R.M.; Benetti, E.; Yamashita, O.M.; Gusmão, G.A. Fitotoxidade de perobamica (Aspidosperma desmanthum) submetidas à deriva de glyphosate. Revista Brasileira de Herbicidas, v.10, n.2, p.103-109, 2011. http:// dx.doi.org/10.7824/rbh.v10i2.108.

Sano, E.E.; Rosa, R.; Brito, J.L.S.; Ferreira, L.G. Land cover mapping of the tropical savanna region in Brazil. Environmental Monitoring and Assessment, v. 166, n.1-4, p.113-124, 2010. http://dx.doi.org/10.1007/s10661-009-0988-4.

Santos, H.G.; Jacomine, P.K.T.; Anjos, L.H.C.; Oliveira, V.A.; Lumbreras, J.F.; Coelho, M.R.; et al. Sistema brasileiro de classificação de solos. 3 . ed. Brasília: Embrapa, 2013. 353p.

Silva, J.B.L.; Sousa, P.A.; Souza, K.B.; Almeida, K.N.S.; Pires, L.C. Evolução temporal do desmatamento na bacia do riacho da Estiva, Piauí. Revista Engenharia na Agricultura, v.23, n.4, p.363-370, 2015. 